\title{
First Principles Calculations of NMR Chemical Shifts of Liquid Water at an Amorphous Silica Interface
}

\author{
By Xiang Yang Guo ${ }^{1}$, Tobias Watermann ${ }^{1}$, Shane Keane ${ }^{1}$, Christoph Allolio ${ }^{1}$, and \\ Daniel Sebastiani ${ }^{1,2, *}$ \\ ${ }^{1}$ Physics Department, Freie Universität Berlin, Arnimalle 14, 14195 Germany \\ ${ }^{2}$ Institute of Chemistry, Martin-Luther-Universität Halle Wittenberg, Kurt-Mothes-Strasse 2, 06120 \\ Halle, Germany
}

Dedicated to Professor Hans Wolfgang Spiess on the occasion of his $70^{\text {th }}$ birthday

(Received June 4, 2012; accepted in revised form September 21, 2012)

(Published online October 29, 2012)

\section{Car-Parrinello Molecular Dynamics Simulations / DFT / NMR Chemical Shift Calculations / MCM-41 / Liquid Water Structure / Confinement}

\begin{abstract}
We investigate the anomalous structure and hydrogen bond network of water molecules confined inside a silica nanopore (MCM-41 type). In addition to geometric data, we use proton NMR chemical shifts as a measure for the strength of the H-bonding network. We compute the ${ }^{1} \mathrm{H}$ NMR shifts of confined water based on a first principle approach in the framework of density functional perturbation theory under periodic boundary conditions. The hydrophilic character of the silica is well manifested in the water density profile. Our calculations illustrate both the modifications of the ${ }^{1} \mathrm{H}$ NMR chemical shifts of the water with respect to bulk water and a considerable slowing down of water diffusion. In the vicinity of silanols, weakly hydrogen bonded liquid water is observed, while at the center region of the pore, the hydrogen bonding network is enhanced with respect to bulk water.
\end{abstract}

\section{Introduction}

Periodically structured porous materials have evoked wide interest for various applications in recent years. Their micro-structure is observed to be composed of orderly arranged pores with uniform size [1]. MCM-41, as one of the most studied type, is characterized by pores less than $40 \AA$ in diameter, within which liquids can be confined. Experimental studies regarding MCM-41 and the properties of liquids confined within it have been performed at length. Techniques such as quasi-elastic and deep elastic neutron scattering [2-4], neutron diffraction with isotopic substitution [5], x-ray spectroscopy [6], sum frequency vibration spectroscopy [7], adsorption calorimetry [8],

\footnotetext{
* Corresponding author. E-mail: daniel.sebastiani@fu-berlin.de
} 
and NMR spectroscopy [9-11] have provided a lot of experimental data which may be compared to simulation data.

The silanol groups on the silica surface play a special role for its ability of supplying hydrogen bonding sites for the confined solvent [14]. With respect to their density on the MCM-41 silica surface $[9,10,13]$, the consensus seems to be about 2 to $3 \mathrm{~nm}^{-2}$, meaning these groups cannot form hydrogen bonds with each other as they are too far apart. Since the average distance between them is about $5.8 \AA$, one water molecule cannot be simultaneously hydrogen bonded to two silanol groups [1]. Up to three water molecules, however, may be hydrogen bonded to a single silanol group [14]. The nature of water differed from bulk water when it is hydrogen bonded to the hydroxyl groups on the silica surface. Recent neutron scattering evidence suggests that the hydrogen bond formed between a water molecule and a silanol in mesoporous silica is stronger than the hydrogen bonds between water molecules [4].

Many simulations have been conducted focusing on the water silica interface. Since the structure of MCM-41 is not fully known, many varying models have been proposed to simulate its surface. For example, Shirono et al. [15] and Kleestorfer et al. [16] used a block of alpha-quartz out of which they cut circular pores of various sizes. In cases where there remained oxygens attached to only one silicon atom, hydrogen atoms were added to create the silanol groups characteristic of the surface of MCM-41. Gallo et al. [12] used beta-cristobalite which was melted and equilibrated at $1000 \mathrm{~K}$. It was then quenched and a cylindrical pore $15 \AA$ in diameter was carved into it. Again, oxygens left attached to only one silicon atom were capped with hydrogen atoms. Sherendovich et al. [9] use different experimentally derived criteria to recommend a model of MCM-41 based on the structure of tridymite. The model they proposed was not in fact tridymite, but a fictitious structure created from pieces of it stuck together in different configurations. With respect to the silica-water interface, Sulpizi et al. [29] conducted ab initio Molecular Dynamics (AIMD) on the hydroxilated (0001) $\alpha$-quartz surface system. Two types of silanol groups were addressed in their results, so called out-of-plane silanols with a strong acidic character and in-plane silanols with weaker acidity.

In this work, a simplified surface model is constructed which has the virtue of containing the same density of silanol groups as MCM-41. We obtain the density and translational mobility profiles of water confined in this model by applying first principle density functional theory based molecular dynamics (AIMD) simulation. Then we compute ensemble averages of Nuclear Magnetic Resonance (NMR) calculations on the confined water molecules and compare the results with bulk water. Furthermore we analyze the distribution of the water NMR shifts along the pore axis and the impact of the geometric confinement on the bound water NMR shifts so as to increase our understanding of the influence of spatial confinement on the structure and dynamics of water.

\section{Computional details}

The water-silica interface is represented by a simplified yet realistic surface model which demonstrates the experimentally known structure of the MCM-41 pore. Figure 1 


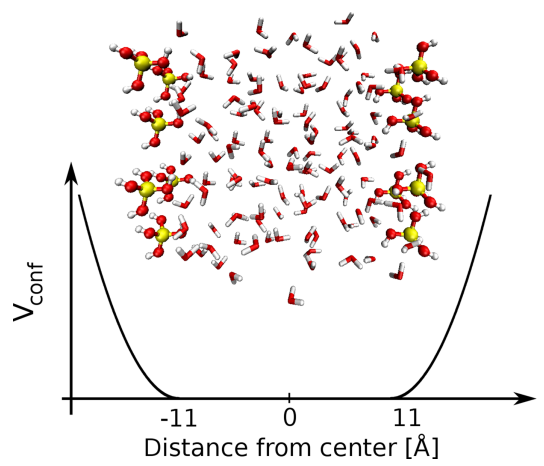

Fig. 1. Two silica-water unit cells from a snapshot of the MD simulation ( $x z$ side view). The threedimensional periodic cell contains six silicic acid groups at the walls and 59 water molecules in between. $\mathrm{Si}, \mathrm{O}$ and $\mathrm{H}$ atoms are represented in yellow, red and white respectively. The silica surface is represented by three isolated $\mathrm{Si}(\mathrm{OH})_{4}$ tetrahedrons.

shows a snapshot of the model. Silicic acid $\mathrm{Si}(\mathrm{OH})_{4}$ is used to model the silanol groups with the three outer hydroxy groups fixed.

Following the experimental results from the NMR spectroscopy $[9,10]$ on the structure of MCM-41, the density of the silanol groups is taken to be $3 \mathrm{~nm}^{-2}$. The possible hydrogen bonding between $\mathrm{Si}-\mathrm{O}-\mathrm{Si}$ bridge and water is missing in this model. We assume it is only of minor influence since the highly concentrated silanol groups on the surface hamper sterically the formation of this type of hydrogen bonds. In the next more sophisticated model, the actual amorphous pore will be considered.

The diffusion of water into the surface is prevented by a harmonic potential (Fig. 1). The potential only acts on the water molecules to allow free $\mathrm{Si}-\mathrm{OH}$ vibrations. The simulation is carried out in a fully periodic box with a size of $28 \times 10 \times 10 \AA$, two flat silanol walls are placed $22 \AA$ apart, $6 \AA$ space is left at either end of the potential to reduce periodic effects in the $x$ direction. 59 water molecules are placed between the two walls. In order to employ a timestep of $1 \mathrm{fs}$, we choose heavy water instead of $\mathrm{H}_{2} \mathrm{O}$ to double the calculation speed.

We run DFT based molecular dynamics simulations in the CP2K package [30]. The BLYP [31] exchange-correlation functional was used, as well as the TZVP basis sets and GTH pseudopotentials [32]. The DFT-D2 Grimme [33] dispersion correction was also used. In total, the simulation ran for over $45 \mathrm{ps}$.

The system was first equilibrated for $10 \mathrm{ps}$ using the canonical ensemble, employing a Nosé-Hoover thermostat. The temperature was set to be $320 \mathrm{~K}$. The simulation was then switched to the microcanonical ensemble for the remaining time.

The NMR chemical shifts are computed as ensemble averages from ab initio nuclear shielding calculations within the CP2K package [30]. A random set of 15 snapshots from the NVE trajectory was sampled, and chemical shifts of all atoms were calculated. For the referencing of the nuclear shielding tensors to chemical shifts, we utilized the method applied in Ref. [23] The NMR simulation used a Gaussian aug- 
mented plane wave approach with GAPW plane wave cutoff 320 Ry, the BLYP-DFT exchange-correlation functional and the TZV2PX-MOLOPT-GTH basis set were employed [34].

It should be noted that we do not compute the quantum propagation of the nuclear spin state which occurs on a timescale of millisecond in a typical NMR experiment, but instead we compute directly the energy difference of the 2 states. Hence the simulation duration on a picosecond level is adequate to achieve the necessary sampling for the averaged instantaneous chemical shifts [35-40].

\section{Results and discussion}

\subsection{Translational dynamical properties}

One of the most salient properties of water confined in MCM-41, discovered by experiments and MD simulations $[12,15,17,18]$ is a slower translational dynamics with respect to bulk water. The translational dynamics of our system were measured by calculating the diffusion coefficient of the system. The ratio of the diffusion coefficient of water confined in MCM-41 to that of bulk water

$$
q=\frac{D_{\mathrm{D}_{2} \mathrm{O}}^{\text {Confined }}}{D_{\mathrm{D}_{2} \mathrm{O}}^{\text {Bul }}}
$$

has been ascertained experimentally and via simulation. Values of $q$ range quite widely from 0.23 to $0.64[15,17,18,24]$, with the consensus showing a much slower diffusion of water within MCM-41 pores. In this work, the reference self diffusion coefficient value for bulk heavy water is taken to be $0.187 \AA^{2} / p s$ from Ref. [28]. In order to obtain the value of $q$ for our system, we calculated the diffusion coefficient $D$ for our confined water according to Einstein's relation

$$
D=\frac{\mathrm{MSD}}{2 d t}=\left\langle\frac{\left(R_{\mathrm{D}_{2} \mathrm{O}}(t)-R_{\mathrm{D}_{2} \mathrm{O}}(0)\right)^{2}}{2 d t}\right\rangle_{\mathrm{D}_{2} \mathrm{O}}
$$

with the numerator representing the mean-square displacement (MSD), $t$ the time, and $d$ the number of dimensions in which the quantity is measured. For the simulation of confined water, only diffusion in the two periodic directions (in our case the $y$ and $z$ dimensions) is taken into account in order to reduce possible distortion due to the presence of the walls [18].

The mean-square displacement (MSD) of water molecules over time in the confined cases is presented in Fig. 2. From this graph, the diffusion coefficient for confined water is calculated to be $0.05 \AA^{2} /$ ps. As $0.187 \AA^{2} /$ ps for bulk water, the resulting ratio $q$ is about 0.27 which falls into the range we obtained from literature.

As pointed out in several studies $[11,12]$, however, taking the diffusion coefficient of the whole system all at once fails to take adequate consideration of different effects of the confinement at different position within the pore. 


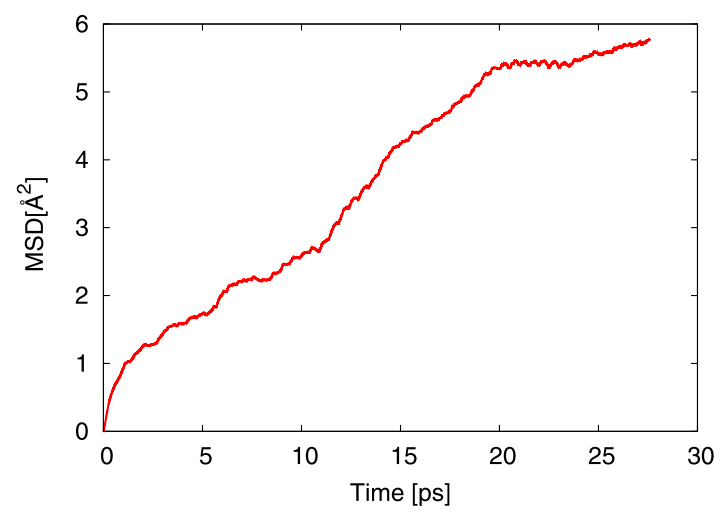

Fig. 2. Global MSD of the oxygen atoms of confined water at $T=320 \mathrm{~K}$.

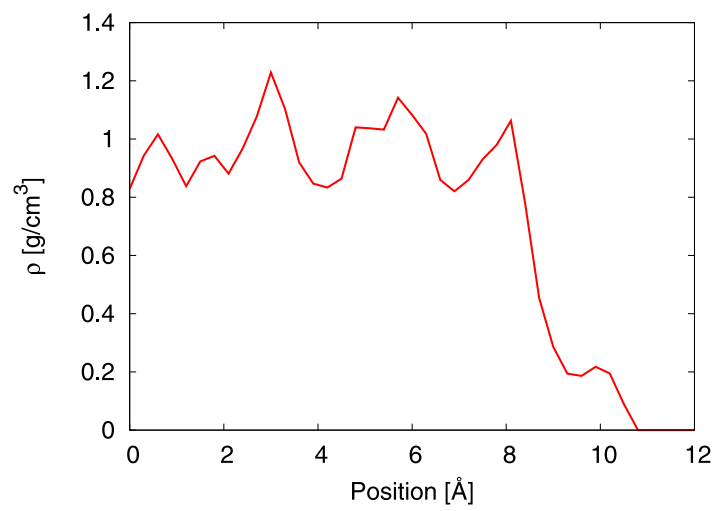

Fig. 3. Density Profile of Water confined between two silica slabs. The origin $x=0 \AA$ corresponds to the center of the pore.

\subsection{Density profile}

The water density profile can demonstrate the configuration of confined water and the hydrophilic character of the silica surfaces. In the earlier empirical structure refinement simulations by R. Mancinelli et al. [5,27], the density of confined water in a MCM-41 pore is found to be higher in the vicinity of the silanol walls; at $300 \mathrm{~K}$ in the interfacial region the density was observed to be about 3 times as large as the density in the middle. This phenomenon stems from a so-called cohesive failure between water molecules. I. e. when water is confined between hydrophilic surfaces, voids occur in the middle of the water layer which leads to cohesive failure [26]. As to the present experimental results, Kocherbitov et al. [8] measured the apparent density of water in the MCM-41 pores at $298 \mathrm{~K}$ to be $0.88 \mathrm{~g} / \mathrm{cm}^{3}$ using $\mathrm{H}_{2} \mathrm{O}$ and $\mathrm{N}_{2}$ sorption method.

In our simulation, the density profile of water was constructed by creating a histogram of the spatial locations of all atoms across the pore with bins $0.3 \AA$ wide and averaging over the entire NVE trajectory, as shown in Fig. 3. The position represents 


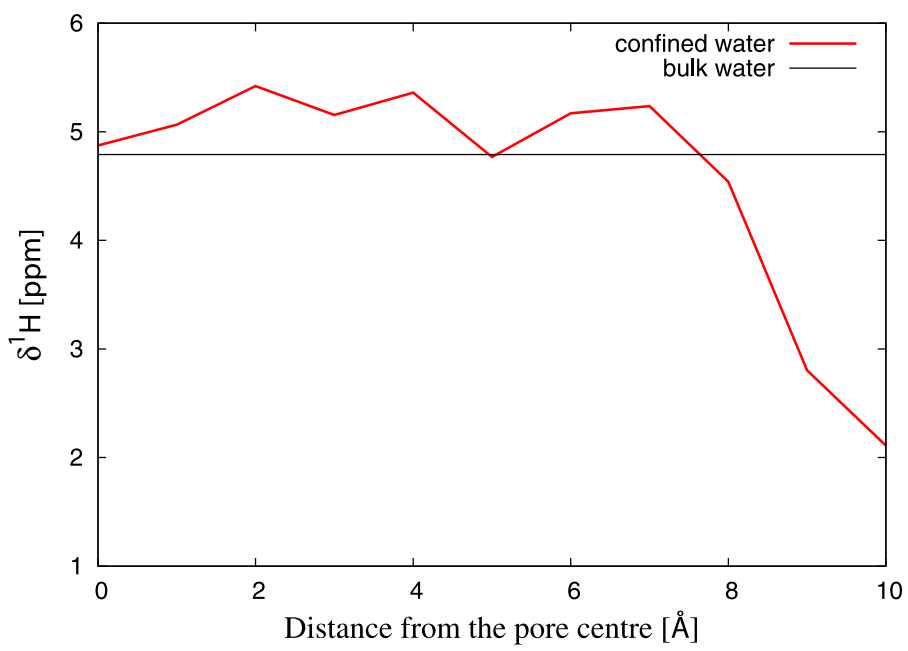

Fig. 4. Calculated ${ }^{1} \mathrm{H}$ NMR chemical shifts of water confined between silica slabs compared with bulk water, the distribution has been symmetrized with respect to the center $(x=0 \AA)$, the value of ${ }^{1} \mathrm{H}$ NMR shifts are averaged in the $x$ direction over the length of the model and the NVE trajectory of the system. The graph is drawn up to where the atoms in $\mathrm{Si}(\mathrm{OH})_{4}$ start to appear.

the distance of the oxygen atoms from the center along the pore axis perpendicular to the silica surface. At the core region of the cell $(0 \AA$ to $2 \AA)$ a lower density appears in our result, and at the outer edge ( $7 \AA$ to $9 \AA$ ) as well, then it gradually decays to zero at the silica wall. Notable density oscillations are observed from Fig. 3, which reveals that the spatial layering occurred in confined water due to the hydrophilic character of the substrate [21]. For example, we see 2 density peaks near the silica substrate, which indicates that there are 2 separate layers of water in this region. However in several other simulations, the produced density profile showed either one [5,18] or two $[12,24,25]$ peaks in the interfacial region and lower density in the core region. The small bump in the density profile near the very edge of the pore is also present in other results $[12,18]$. One possible reason for the minimum appearing here is the presence of the silanol groups oxygen atoms in that area taking up space.

\section{3 ${ }^{1} \mathrm{H}$ NMR chemical shift calculations}

Lately the $a b$ initio calculation of nuclear chemical shifts has become one of the most powerful methods for structure determination on the molecular level. Not merely can the calculation interpret the spectra returned by the experiments [23], but also it can produce instantaneous results that are beyond experimental capability. Particularly the instantaneous ${ }^{1} \mathrm{H}$ NMR shifts can provide significant probe for the hydrogen bonding network of specific chemical environments [41,42].

The NMR shifts profile we obtained, as shown in Fig. 4, substantially indicates the configuration of water confined between the 2 silica slabs. The experimental ${ }^{1} \mathrm{H}$ NMR chemical shift $\delta=4.79 \mathrm{ppm}$ for bulk water is taken from the value given in Ref. [22]. Figure 4 displays that in the major part of the cell, within $7.6 \AA$ away from the center, 
stronger H-bonded water appears with $\delta$ varying from $4.7 \mathrm{ppm}$ to $5.4 \mathrm{ppm}$. The largest $\delta=5.4 \mathrm{ppm}$ is reached at $x=2 \AA$. At the core of the pore $(x=0 \AA), \delta=4.86 \mathrm{ppm}$. Due to the confining effects of silica slabs, the NMR shifts are greater than those of bulk water. But at the core of the pore, the calculated shift approximates that of the bulk water, reflecting the hydrogen bonding in this region resembles bulk water H-bond network. While the shifts drop to upper-field as approaching the wall, in the vicinity of silanol walls (from $x=7.6 \AA$ to $x=10 \AA$ ), water molecules are found to form weaker hydrogen bonds with each other or with silanols, the minimum value $2.1 \mathrm{ppm}$ is found at $x=10 \AA$. As discussed before, the density profile shows layering effect took place in our cell. According to Gallo et al. [21], the interactions of the substrate atoms and thin water layers causes a strong distortion of the H-bond network. This explains why we see a decline of the shifts near the wall, even though it has been experimentally proven that the single silanol-water $\mathrm{H}$-bond is stronger than the water-water $\mathrm{H}$-bond [4].

We analogize the water filling process inside the pore by calculating the running average of ${ }^{1} \mathrm{H}$ chemical shifts up to a certain distance from the wall using equation

$$
\delta_{\mathrm{Avg}}{ }^{1} \mathrm{H}(x)=\frac{1}{N_{\mathrm{H}}} \sum_{x^{\prime}=x_{\text {wall }}}^{x} \delta^{1} \mathrm{H}(x)
$$

$\delta_{\mathrm{Avg}}{ }^{1} \mathrm{H}(x)$ represents the average $\delta$ value of a fictitious water film with thickness $x$. For each value of $\delta_{\mathrm{Avg}}{ }^{1} \mathrm{H}(x)$, we sampled from the wall to the plane at corresponding distance and calculated all the shifts of water between the 2 planes. Taking the limitation of sampling into account, we leave out the first averaged value from $x=0 \AA$ to $0.5 \AA$. The range of averaged NMR shifts along the $x$ direction over the length of the cell covers from 2 to $4.9 \mathrm{ppm}$ which is in good agreement with the experimental value from 1.74 to 4.7 ppm achieved in Buntkowsky's group [1]. Grünberg et al. obtained the chemical shift spectra of confined water at varying hydration level using ${ }^{1} \mathrm{H}$ solid-state NMR. In their results for the fully hydrated pore, one single signal appears at $4.7 \mathrm{ppm}$, and with very small water content $(\leq 2.7 \%)$, one dominating peak locates at $1.74 \mathrm{ppm}$ which is ascribed to the shift of silanol groups.

Figure 5 shows that the behavior of water in our model resembles the water filling process described in Ref. [1]. Comparing our data with the experimental findings, we observe the same varying tendency in the chemical shifts. The minimum value of $\delta$ in Fig. 5 was obtained by computing the shifts of water locating within $0.5 \AA$ to the silica substrate, so as to correspond the very low water filling factor in the experiments. In the interfacial region,the experimental NMR shift is an approximation to the shift of silanol groups. Therefore, it is believed that all water molecules at this region were hydrogen bonded to the silanols. With increased water content, additional water molecules start to form hydrogen bonds with each other which gives rise to ascending chemical shifts. This phenomenon is also reflected in Fig. 5, but increasing distance from the wall plays the role instead of increasing water filling factor. When the water content goes up to $3.2 \%$, a notable peak at $2.5 \mathrm{ppm}$ arises beside a weakened line at $1.74 \mathrm{ppm}$, which indicates the coexistence of 2 different types of hydrogen bonds. The NMR peak shifts consistently towards down-field upon further increasing hydration level. When there is $23 \%$ of water, the line at $2.5 \mathrm{ppm}$ is broadened and shifts to $3.4 \mathrm{ppm}$. When the pore is completely filled, a single shift appears at $4.7 \mathrm{ppm}$. 


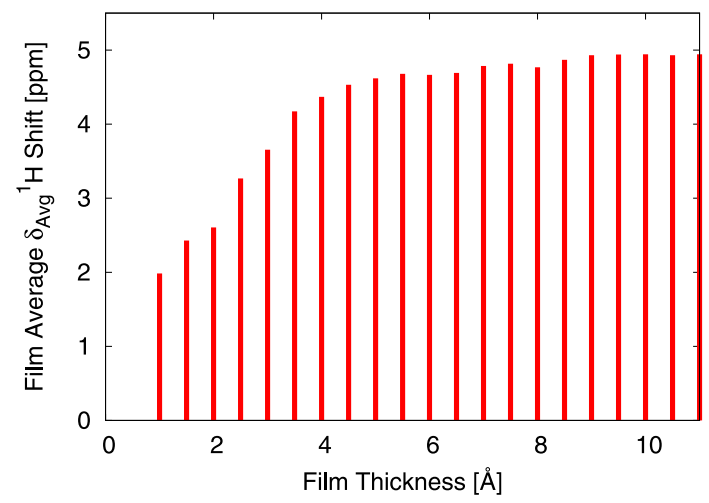

Fig. 5. Average of the ${ }^{1} \mathrm{H}$ NMR chemical shift values of those water molecules residing within a film of given thickness from the wall. The film thickness axis $0 \AA$ and $11 \AA$ are corresponding to the wall and the cell center respectively.

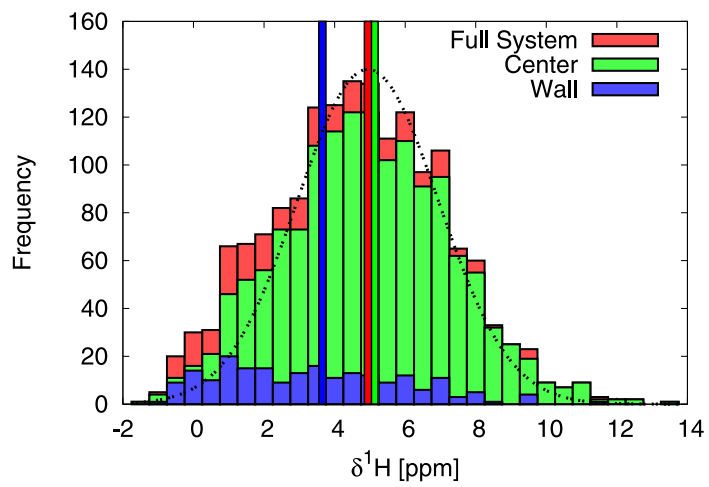

Fig. 6. Calculated ${ }^{1} \mathrm{H}$ NMR shift distribution of confined water. The ordinate is the occurence of the shifts for various $\delta$ values. $\delta=3.65 \mathrm{ppm}$ is the averaged value for the water within $3 \AA$ proximity to the walls (blue), $\delta=4.94 \mathrm{ppm}$ is the global average of the whole system (red), and $\delta=5.13 \mathrm{ppm}$ is the average shift of the water at the center of the pore (green). The dashed curve represents a gaussian centered at $\delta=4.94$ ppm (black).

Our averaged NMR shifts imitate this process (as shown in Fig. 5) in such a way that the $\delta$ value continually goes up from $2 \mathrm{ppm}$ to $5.5 \mathrm{ppm}$ as increasing thickness of the film which starts from the wall $(0 \AA)$. This denotes that the bonding between water and the silanols becomes less and less dominating which leads to the the growing of the shifts until $x=5.5 \AA$. A plateau arises at $5.5 \AA<x<11 \AA$ reflecting the water molecules at the center region are mostly bounded to each other and not influenced by the walls.

Figure 6 illustrates the spacial distribution of the ${ }^{1} \mathrm{H}$ NMR shifts inside the cell. Those water molecules situated within $3 \AA$ proximity to the silica wall are denoted as wall water, and the rest are considered to be center water. In this way, the confined water is divided into 3 layers, silanol-water interface (wall water), water-water (center 
water) and water-silanol interface (wall water). The computed global average value of the ${ }^{1} \mathrm{H}$ chemical shift for all the water inside this cell is $4.94 \mathrm{ppm}$, the averaged water shift in the close region of walls is $3.65 \mathrm{ppm}$, and that for the water in the core area is $5.13 \mathrm{ppm}$. Comparing the whole distribution with a Gaussian centered at the global average $\delta$ value $4.94 \mathrm{ppm}$, we can easily see that more water molecules fall into the up field in the proximity of silanols. Most wall water resides at the left half of the graph with $\delta \leq 4.94 \mathrm{ppm}$. The overall average shift of the system moves towards lower $\delta$ (upper field) under the effects of silica walls. This gives further evidence that the silanol groups play a twisting role for the water-water hydrogen bonding network in the interfacial region [21].

\section{Conclusion}

In this paper, DFT based molecular dynamics simulations and first principles NMR chemical shift calculations are applied on a simplified surface model based on the structural properties of the MCM-41 pore. Our calculations demonstrate the strong influence of spatial confinement on the structure and dynamical properties of water.

On the structural level, we see a strong influence on the ${ }^{1} \mathrm{H}$ chemical shifts of the confined water. While at the center of the pore a $0.5 \mathrm{ppm}$ increased chemical shift compared to bulk water is found, the chemical shift gradually decreases when approaching the wall, until it reaches a value 3 ppm below bulk water reference. This implies an enhanced hydrogen bonding network for the water at the center, and a strongly weakened network close to the silica-water interface. In the density profile, distinctive peaks appear next to the wall, indicating a structuring of the water by the wall geometry. By calculating average chemical shifts for fictitious water films at the wall, we can see a change from low chemical shifts for thin films towards bulk water like chemical shifts for a film thickness above $4 \AA$. This is in good agreement with a previous experimental model on the gradual filling of nanopores.

With respect to the dynamical behavior, we see a decrease in the diffusion rate by a factor of 4 when comparing to bulk water values.

\section{Acknowledgement}

This work has been supported by the German Research Foundation (DFG) under grants SE 1008/5 and 1008/8. Computing infrastructure was provided by the Northern German Supercomputing Alliance (HLRN) under grant HLRN/bec00073 and ZEDAT high-performance cluster in Freie Universität Berlin. We thank the German academic exchange service (DAAD) for a RISE internship.

\section{References}

1. B. Grünberg, T. Emmler, E. Gedat, I. Shenderovich, G. H. Findenegg, H. H. Limbach, and G. Buntkowsky, Chem. Eur. J. 10 (2004) 5689.

2. A. Faraone, L. Liu, C. Mou, P. Shih, J. Copley, and S. Chen, J. Chem. Phys. 7 (2003) 3963.

3. F. Mansour, R. M. Dimeo, and H. Peemoeller, Phys. Rev. E 66 (2002) 041307. 
4. C. Pantalei, R. Senesi, C. Andreani, P. Sozzani, A. Comotti, S. Bracco, M. Beretta, Mario, P. Sokol, and G. Reiter, Phys. Chem. Chem. Phys. 13 (2011) 6022.

5. R. Mancinelli, S. Imberti, A. K. Soper, K. H. Liu, C. Y. Mou, F. Bruni, and M. A. Ricci, J. Phys. Chem. B 113 (2009) 16169.

6. P. Smirnov, T. Yamaguchi, S. Kittaka, S. Takahara, and Y. Kuroda, J. Phys. Chem. B 104 (2000) 5498.

7. V. Ostroverkhov, G. A. Waychunas, and Y. R. Shen, Phys. Rev. Lett. 94 (2005) 046102.

8. V. Kocherbitov and V. Alfredsson, J. Phys. Chem. B 112 (2007) 12906.

9. I. G. Shenderovich, D. Mauder, D. Akcakayiran, G. Buntkowsky, H. H. Limbach, and G. Findenegg, J. Chem. Phys. B 111 (2007) 12088.

10. X. S. Zhao, G. Q. Lu, A. K. Whittaker, G. J. Millar, H. Y. Zhu, J. Chem. Phys. B 101 (1997) 6525.

11. D. W. Hwang, A. K. Sinha, C. Y. Cheng, T. Y. Yu, and L. P. Hwang, J. Phys. Chem. B 105 (2001) 5713.

12. P. Gallo, M. Rovere, and S. H. Chen, J. Phys.: Condens. Matter 24 (2012) 064109.

13. S. Pizzanelli, S. Kababya, V. Frydman, M. Landau, and S. Vega, J. Phys. Chem. B 109 (2005) 8029.

14. E. P. Ng and S. Mintova, Micropor. Mesopor. Mat. 14 (2008) 1.

15. K. Shirono and H. Daiguji, J. Phys. Chem. B 111 (2007) 7938.

16. K. Kleestorfer, H. Vinek, and A. Jentys, J. Mol. Catal. A: Chem. 166 (2001) 53.

17. S. Takahara, N. Sumiyama, S. Kittaka, T. Yamaguchi, B. Funel, and M. Claire, Adsorption 109 (2005) 5814.

18. A. Lerbret, G. Lelong, P. Mason, M. L. Saboungi, and J. Brady, Food Biophys. 6 (2011) 233.

19. M. E. Tuckerman, Statistical Mechanics: Theory and Molecular Simulation, Oxford Graduate Texts, Oxford University Press Inc., New York (2010).

20. A. R. Bizzarriand and S. Cannistraro, J. Phys. Chem. B 106 (2002) 6617.

21. P. Gallo, M. Rapinesi, and M. Rovere, J. Chem. Phys. 117 (2002) 7.

22. H. E. Gottlieb, V. Kotlyar, and N. Abraham, J. Org. Chem. 62 (1997) 7512.

23. D. R. Banyai, T. Murakhtina, and D. Sebastiani, Magn. Reson. Chem. 48 (2010) S56.

24. P. A. Bonnaud, B. Coasne, and R. J-M. Pellenq, J. Phys.-Condens. Mat. 22 (2010) 284110.

25. A. A. Milischuk and B. M. Ladanyi, J. Chem. Phys. 135 (2011) 174709.

26. T. G. Lombardo, N. Giovambattista, and P. G. Debenedetti, Faraday Discuss. 141 (2009) 359.

27. R. Mancinelli, F. Bruni, and M. A. Ricci, J. Phys. Chem. Lett. 1 (2010) 1277.

28. R. Mills, J. Phys. Chem. 77 (1973) 685.

29. M. Sulpizi, M. Gaigeot, and M. Sprik, J. Chem. Theory 8 (2012) 1037.

30. Computer code CP2K, available from www: www.cp2k.org/.

31. A. D. Becke, Phys. Rev. A 38 (1988) 3098.

32. S. Goedecker, M. Teter, and J. Hutter, Phys. Rev. B 54 (1996) 1703.

33. S. Grimme, J. Comput. Chem. 27 (2006) 1787.

34. J. VandeVondele and J. Hutter, J. Chem. Phys. 127 (2007) 114105.

35. D. Sebastiani and M. Parrinello, Chem. Phys. Chem. 3 (2002) 675

36. J. Schmidt, J. VandeVondele, I. F. Kuo, D. Sebastiani, J. I. Siepmann, J. Hutter, and C. J. Mundy, J. Phys. Chem. B 113 (2009) 11959.

37. J. Schmidt, A. Hoffmann, H. W. Spiess, and D. Sebastiani, J. Phys. Chem. B 110 (2006) 23204.

38. T. Murakhtina, J. Heuft, J. E. Meijer, and D. Sebastiani, Chem. Phys. Chem. 7 (2006) 2578.

39. Y. J. Lee, T. Murakhtina, D. Sebastiani, and H. W. Spiess, J. Am. Chem. Soc. 129 (2007) 12406.

40. A. Hoffmann, D. Sebastiani, E. Sugiono, K. S. Kim, H. W. Spiess, and I. Schnell, Chin. Phys. Lett. 388 (2004) 164.

41. K. Münnemann and H. W. Spiess, Nature Phys. 7 (2011) 522.

42. T. Metzroth, A. Hoffmann, R. Martín-Rapún, M. M. J. Smulders, K. Pieterse, A. R. A. Palmans, J. A. J. M. Vekemans, E. W. Meijer, H. W. Spiess, and J. Gauss, Chem. Sci. 2 (2011) 69. 